\title{
Static Characterization of the Birefringence Effect in the Semiconductor Optical Amplifier Using the Finite Difference Method
}

\author{
A. Elyamani*, A. Zatni* ${ }^{* *}$ H. Bousseta ${ }^{*}$, A. Moumen ${ }^{*}$ \\ ${ }^{*}$ M.S.I.T Laboratory, Department of Computer Engineering high school of technology, Ibn Zohr University Agadir \\ Morocco. \\ ** Department of Computer Engineering, high school of technology, Agadir Morocco
}

\begin{tabular}{l} 
Article Info \\
\hline Article history: \\
Received Oct 16, 2014 \\
Revised Nov 18, 2014 \\
Accepted Dec 12, 2014 \\
\hline Keyword: \\
Birefringence effect \\
Finite difference method \\
(FDM) \\
Non-linearity \\
Polarisation rotation \\
Semiconductor optical \\
amplifier (SOA)
\end{tabular}

\begin{abstract}
Knowing the various physical mechanisms of the semiconductor optical amplifier (SOA) helps us to develop a more complete numerical model. It also enables us to simulate more realistically the static behavior of the $\mathrm{SOA}_{\mathrm{s}}$ ' birefringence effect. This way, it allows us to study more precisely the behavior of $\mathrm{SOA}_{\mathrm{s}}$, and particularly the impact of the amplified spontaneous emission (ASE) or the pump and probe signals as well as the optical functions based on the non-linearity of the component. In static regime, the $\mathrm{SOA}_{\mathrm{s}}$ possess a very low amplification threshold and a saturation power of the gain which mainly depends on the optical power injected into the active region. Beyond the optical input power, the SOA is in the saturated gain regime which gives it a nonlinear transmission behavior. Our detailed numerical model offers a set of equations and an algorithm that predict their behavior. The equations form a theoretical base from which we have coded our model in several files.cpp that the Language C++ executes. It has enabled us, from the physical and geometrical parameters of the component, to recover all the relevant values for a comprehensive study of $\mathrm{SOA}_{\mathrm{s}}$ in static and dynamic regimes. In this paper, we propose to make a static characterization of the effect of the nonlinear polarization rotation by realizing a pump-probe assemblage to control the power and state of polarization at the entering of the SOA.
\end{abstract}

Copyright (C) 2015 Institute of Advanced Engineering and Science. All rights reserved.

Corresponding Author:

A. Elyamani,

M.S.I.T Laboratory, Department of Computer Engineering high school of technology, Ibnou Zohr University Agadir, Morocco

Email: abdenbi.elyamani@gmail.com

\section{INTRODUCTION}

In recent years, there has been considerable progress in the exploitation of optical nonlinearities in $\mathrm{SOA}_{\mathrm{s}}$ [1], [2]. Much attention has been paid to the birefringence in SOA in a pump-probe assemblage. The effective refractive indices for transverse-electric (TE) mode and transverse-magnetic (TM) mode are different form each other due to intrinsic birefringence in SOA and induced birefringence in SOA, thus the phase changes in SOA for TE and TM modes. The origin of polarization-dependent gain (PDG) in $\mathrm{SOA}_{\mathrm{S}}$ is the fact that bulk active material has much larger TE amplification than TM, which is due to the different confinement factors [3]. In addition to the cumulative effect of PDG, the SOA $\mathrm{A}_{\mathrm{s}}$ also introduce ASE noise, which affects the optical signal-to-noise ratio (OSNR) of the payload channels.

The theoretical foundation of SOAs modeling was established in 1980s. Since then, major progress concerning SOA modeling has started to focus on the material gain coefficient, spontaneous emission rate and the fraction of spontaneous emissions coupled with the guided waves in an amplifier. Some numerical 
simulations which use the Finite Difference Method (FDM) [4] have been studied intensively to solve carrier rate equation and photon traveling wave equations [5], [6].

In this paper, we propose to make a static characterization of the effect of the nonlinear polarization rotation by realizing a pump-probe assemblage to control the power and state of polarization at the entering of the SOA. Therefore, this paper is dedicated to the study of modifications to model suggested by Connelly to take into account the effect of birefringence in the SOA. The expressions of the coupled mode equations must be modified. We have developed a more detailed model based on the equations of the coupled modes which depend on the polarization and use the finite difference method (FDM). This model treats separately the TE component and TM component of the optical field, introduces different confinement factors for the two polarization states and takes into account the phenomenon of the TE and TM modes energy coupling. The results we have obtained provide an instructive insight into SOA. These results are also beneficial for device design and optimization.

\section{THEORY OF SOA}

\subsection{Theoretical Model}

We decompose the incoming arbitrarily polarized electric field in a TE component and TM component as illustrated in figure 1. These two polarization directions are along the principal axes $(x, y)$ that diagonalize the wave propagation in the SOA.

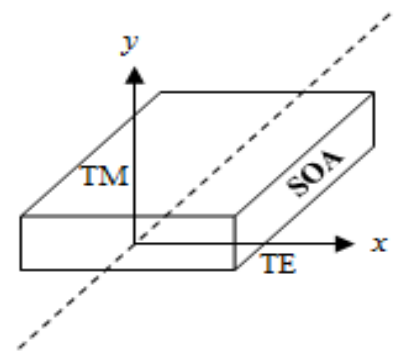

Figure 1. A SOA and the two polarization directions TE and TM schema

The total electric field is defined by [1]:

$$
\vec{E}=E_{T E} \vec{x}+E_{T M} \vec{y}
$$

With: $\quad\left\{\begin{array}{l}E_{T E}=E_{0} \times \operatorname{COS}(\theta) \times \exp \left(j \emptyset_{x}\right) \\ E_{T M}=E_{0} \times \operatorname{COS}(\theta) \times \exp \left(j \emptyset_{y}\right)\end{array}\right.$

Whereas $\theta$ is the input polarization angle, $\emptyset_{x}$ and $\emptyset_{y}$ are initial phase of input signal for TE mode and TM mode, respectively. In our calculation, in left (input) and right (output) facets of SOA have power reflectivities $R_{1}$, and $R_{2}$, respectively. The signal wave gets partially transmitted and reflected from the two facets of the amplifier. Due to the input signal, the spatially varying component of the field in the SOA can be decomposed into forward and backward propagating $\mathrm{E}^{+}, \mathrm{E}^{-}$, and the traveling-waving equation for TE mode and TM mode are [1], [7], [8] and [9]:

$$
\begin{aligned}
& \left\{\begin{array}{l}
\frac{d E_{k, T E}^{+}(z)}{d z}=+\left\{\left(-j \beta_{k, T E}(n)+\frac{1}{2} g_{n, T E}\left(\vartheta_{k}, n\right)\right) E_{k, T E}^{+}(z)+j C_{k, T M-T E}(n) E_{k, T M}^{+}(z)\right\} \\
\frac{d E_{k, T E}^{-}(z)}{d z}=-\left\{\left(-j \beta_{k, T E}(n)+\frac{1}{2} g_{n, T E}\left(\vartheta_{k}, n\right)\right) E_{k, T E}^{-}(z)+j C_{k, T M-T E}^{*}(n) E_{k, T M}^{-}(z)\right\}
\end{array}\right. \\
& \left\{\begin{array}{l}
\frac{d E_{k, T M}^{+}(z)}{d z}=+\left\{\left(-j \beta_{k, T M}(n)+\frac{1}{2} g_{n, T M}\left(\vartheta_{k}, n\right)\right) E_{k, T M}^{+}(z)+j C_{k, T E-T M}(n) E_{k, T E}^{+}(z)\right\} \\
\frac{d E_{k, T M}^{-}(z)}{d z}=-\left\{\left(-j \beta_{k, T M}(n)+\frac{1}{2} g_{n, T M}\left(\vartheta_{k}, n\right)\right) E_{k, T M}^{-}(z)+j C_{k, T E-T M}^{*}(n) E_{k, T E}^{-}(z)\right\}
\end{array}\right.
\end{aligned}
$$

Those spontaneous emission photon rates are observed in the following equation:

Static Characterization of the Birefringence Effect in the Semiconductor Optical Amplifier ... (A Elyamani) 


$$
\begin{aligned}
& \left\{\begin{array}{l}
\frac{d N_{j, T E}^{+}(z)}{d z}=+\left\{g_{n, T E}\left(\vartheta_{j}, n\right) N_{j, T E}^{+}(z)+R_{s p, T E}\left(\vartheta_{j}, n\right)\right\} \\
\frac{d N_{j, T E}^{-}(z)}{d z}=-\left\{g_{n, T E}\left(\vartheta_{j}, n\right) N_{j, T E}^{-}(z)+R_{s p, T E}\left(\vartheta_{j}, n\right)\right\}
\end{array}\right. \\
& \left\{\begin{array}{l}
\frac{d N_{j, T M}^{+}(z)}{d z}=+\left\{g_{n, T M}\left(\vartheta_{j}, n\right) N_{j, T M}^{+}(z)+R_{s p, T M}\left(\vartheta_{j}, n\right)\right\} \\
\frac{d N_{j, T M}^{-}(z)}{d z}=-\left\{g_{n, T M}\left(\vartheta_{j}, n\right) N_{j, T M}^{-}(z)+R_{s p, T M}\left(\vartheta_{j}, n\right)\right\}
\end{array}\right.
\end{aligned}
$$

Whereas $j=\sqrt{-1}, C_{k, T M-T E}$ and $C_{k, T E-T M}$ are the coefficients of coupling modes TE-TM and TM-TE respectively.

$$
\left\{\begin{array}{l}
C_{k, T E-T M}(n)=k_{r} \times \exp \left(j\left(\beta_{k, T M}(n)-\beta_{k, T E}(n)\right) z\right) \\
C_{k, T M-T E}(n)=k_{r} \times \exp \left(j\left(\beta_{k, T E}(n)-\beta_{k, T M}(n)\right) z\right)
\end{array}\right.
$$

Whereas $k_{r}$ is the coupling constant [10], the star represents the complex conjugate. The other coefficients are defined in [5], [7].

The developed model based on the assumption of quasi-stationary is summed up in a several section division of the component gain region so as to take into account the non uniform distribution of carrier density and refractive index. The carrier density in section $i$ inside the SOA obeys the rate equation [7]:

$$
\frac{d n(z, t)}{d t}=\frac{I}{e d w L}-R(n)-\frac{\Gamma_{T E}}{d w}\left(f_{k, T E}(n)-2 f_{j, T E}(n)\right)-\frac{\Gamma_{T M}}{d w}\left(f_{k, T M}(n)-2 f_{j, T M}(n)\right)
$$

Whereas $I$ is the amplifier bias current, $d$ and $w$ are the active region thickness and width, respectively.

The recombination rate term $R(n)$ is given by:

$$
R(n)=C_{1} \times n+C_{2} \times n^{2}+C_{3} \times n^{3}
$$

Whereas $C_{1}, C_{2}$ and $C_{3}$ are recombination coefficients.

$f_{k, T E}, f_{k, T M}, f_{j, T E}$ and $f_{j, T M}$ are defined by equations [7], [9]:

$$
\begin{aligned}
& f_{k, T E}(n)=\sum_{k=1}^{N_{s}} g_{m, T E}\left(\vartheta_{k}, n\right)\left[\left|E_{k, T E}^{+}\right|^{2}+\left|E_{k, T E}^{-}\right|^{2}\right] \\
& f_{k, T M}(n)=\sum_{k=1}^{N_{S}} g_{m, T M}\left(\vartheta_{k}, n\right)\left[\left|E_{k, T M}^{+}\right|^{2}+\left|E_{k, T M}^{-}\right|^{2}\right] \\
& f_{j, T E}(n)=\sum_{j=0}^{N_{m}-1} g_{m, T E}\left(\vartheta_{j}, n\right)\left[N_{j, T E}^{+}+N_{j, T E}^{-}\right] \\
& f_{j, T M}(n)=\sum_{j=0}^{N_{m}-1} g_{m, T M}\left(\vartheta_{j}, n\right)\left[N_{j, T M}^{+}+N_{j, T M}^{-}\right]
\end{aligned}
$$

SOA geometrical and material parameters used in the steady-state model are given in table I.

\subsection{Polarization-Dependent Gain (PDG)}

The dependence of SOA to polarization is characterized by measuring the polarization-dependent gain (PDG). In practice this consists of measuring the gain of the device in all possible cases of polarization signals. To simplify our studies, we have simply sought two orthogonal axes of linear polarization (TE and TM axes). The gain hasn't, therefore, been measured but for the polarized signals along these two axes. The polarization-dependent gain can be calculated via the following formula:

$$
P D G=\left|G_{T E}-G_{T M}\right| \quad(d B)
$$

Two cases of measuring the PDG can then be presented: configurations in self-saturation and crosssaturation. The measurement of the PDG in the self-saturation enables us to inquire on the intrinsic 
dependence of the SOA component which is a very important measure. The measures of the PDG in the configuration pump-probe allow studying the optical gates which operate in cross-modulation.

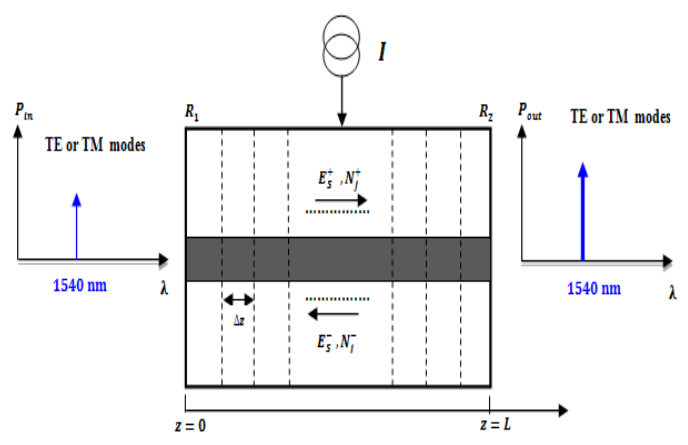

(a)

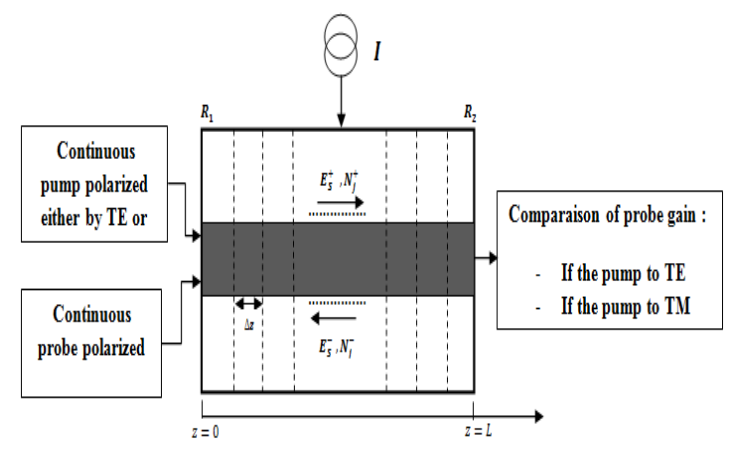

(b)

Figure 2. Schematic view of a typical SOA structure to measure the static gain and the PDG: (a) configuration self-saturation; (b) configuration cross-saturation (pump-probe).

Figure 2 schematizes the principle of these two measures. In each measure, the PDG of the device is evaluated by comparing the curves of static gain obtained with a continuous pump, according to the two extreme configurations of polarization (TE and TM). The greater the difference between the two obtained TE and TM curves is, the higher the PDG.

Table I. SOA geometrical and material parameters [1], [7]

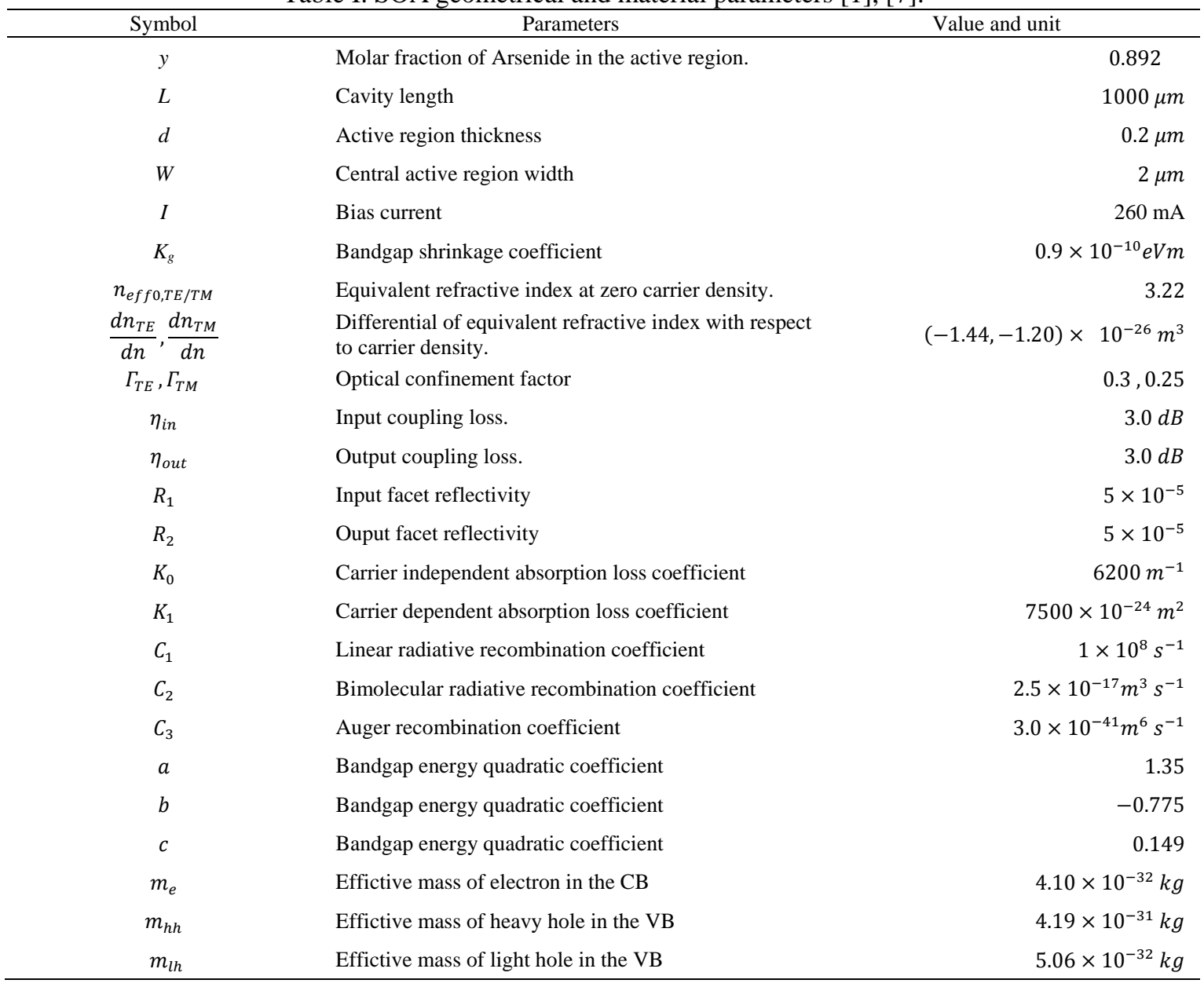

Static Characterization of the Birefringence Effect in the Semiconductor Optical Amplifier ... (A Elyamani) 


\section{RESULTS AND DISCUSION}

The measure of static gain consists of measuring the amplification of a signal as it passes through a SOA depending on the power of the input signal. There are two types of measures: self-gain saturation and cross-gain saturation or pump-probe. The first measure involves injecting a single signal in the SOA (figure .2 (a)) whereas the second one involves two signals called pump and probe.

\subsection{Self-Gain Saturation}

The measure of self-gain saturation of the SOA takes into account the ASE noise. Indeed, in the case of a low optical injection, the ASE power constitutes a considerable additive noise disturbing the detection of the amplified signal. It is necessary to measure the gain by removing the ASE contribution.

The shape of the above curve (figure 3) has two zones: a zone where the gain is constant, called small signal regime, and an area where the gain falls linearly, called saturation regime. While the first zone allows to measure the small signal gain $\mathrm{G}_{0}$, the second one measures that of the saturation power at $-3 \mathrm{~dB}$ $\left(\mathrm{P}_{\text {sat, in }}\right)$.

It should also be noted that the higher the bias current of SOA is, the more available carriers for electron-hole recombination there are, particularly for stimulated emission, and thus, the higher the small signal gain is. In addition, the more the current is, the more important the ASE. Thus, the SOA saturation power is low [5] (The ASE participates in the gain saturation).

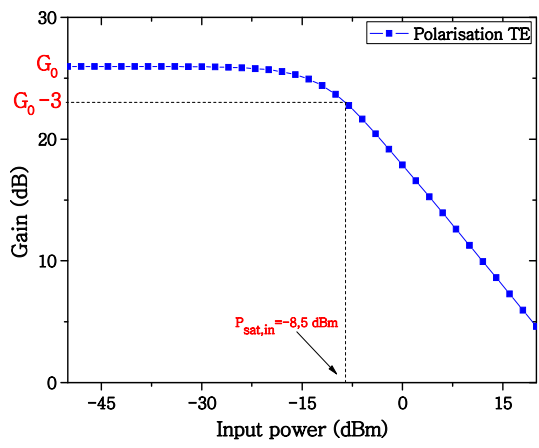

Figure 3. Static gain of the SOA in self-saturation

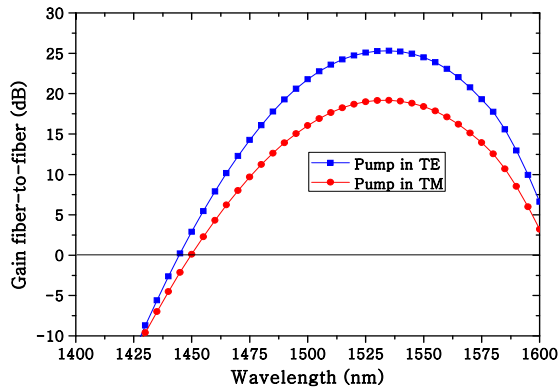

(a)

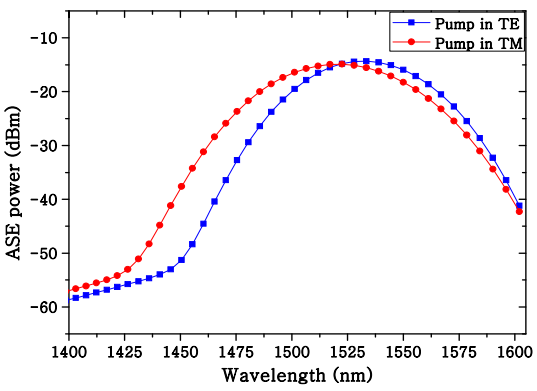

(b)

Figure 4. Gain and ASE spectra according to the TE and TM polarization for Pin $=-20 \mathrm{dBm}$.

Figure 4 presents the gain and ASE spectra solved for the TE and TM polarization. We note effectively a transparency and peak wavelength difference as well as a difference between maxima. This means that the SOA used has some intrinsic polarization dependence. According to the SOA $\mathrm{A}_{\mathrm{s}}$ geometry and the gain and ASE spectra, this tendency comes from the elastics contraints applied to the SOA which have largely favored the TE transitions.

We then studied the PDG of the SOA in self-saturation. For this, we have used the figure schematized above (figure 2 (a)). 
The result obtained (Fig .5) shows that the SOA presents a PDG in self-saturation of the order of 1 $\mathrm{dB}$ in small signal regime. The PDG is subsequently increasingly low when entering in saturation regime. This is explained by the difference in gain due to preferred transitions TE or TM between conduction and valence bands. Experiencing low pumping, the bands are not full, so there is a difference in distribution between the carrier TE and TM. At high pumping, all the bands are saturated, the distribution is equal and the anisotropy saturates. These curves are thus characterized by small signal gains TE and TM different from each other and saturation power TE and TM different from one another as well. These differences are more precisely noted below:

$$
\left\{\begin{array}{c}
G_{0}^{T E}>G_{0}^{T M} \\
P_{\text {sat }}^{T E}(\approx-8.5 \mathrm{dBm})<P_{\text {sat }}^{T M}(\approx-6 \mathrm{dBm})
\end{array}\right.
$$

The SOA used in this study is intrinsically polarization-dependent.

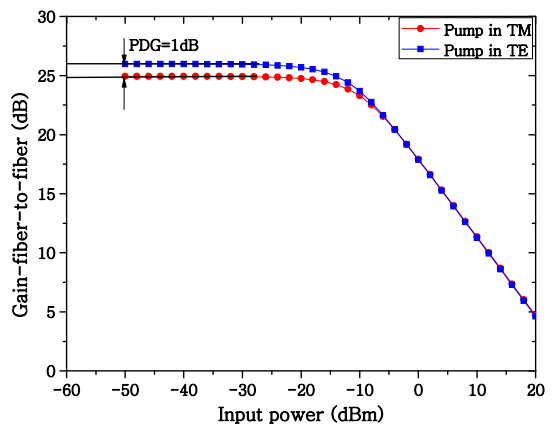

Figure 5. The PDG of SOA in self-saturation.

\subsection{Cross-Gain Saturation}

This measure consists of injecting two continuous signals in the SOA, one of fixed power, the probe, whose gain is measured depending on the power of the other signal, the pump. Figure 2 (b) shows the scheme of assemblage of such a characterization.

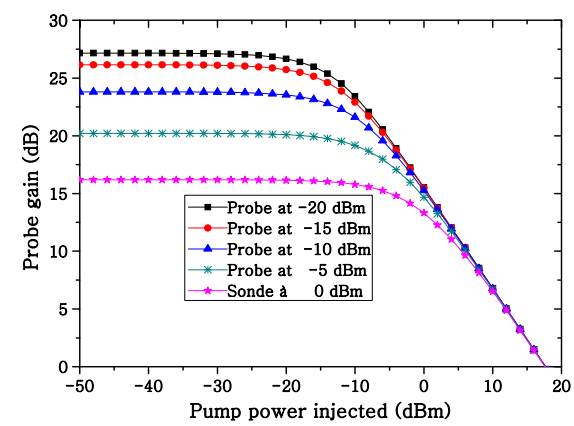

Figure 6. Static gain in pump-probe for different powers of probes. The pump and probe are TE polarized

An example of curves obtained with the same SOA but for different probe powers is reported in figure 6 . The curves present an identical form to that of self-gain saturation. Indeed, be it traversed by one or more signals, the SOA has two operating regimes: the small-signal gain regime and the saturation regime. These curves are, however, different according to the power of the probe signal. This results from the fact that the probe signal may also, according to its power, saturates the SOA, and thus participates in the depopulation of the conduction band, leading to a small signal gain generally lower. In addition less porters, in this case, participate in amplified spontaneous emission (ASE), which leads to an increase of the saturation power of the SOA. Note also that the higher the power probe is, the lower the slope of the curve is and thus less efficient compression gain by a pump pulse is. Finally, for high power pump, the curves meet showing

Static Characterization of the Birefringence Effect in the Semiconductor Optical Amplifier ... (A Elyamani) 
that the gain saturation depends intrinsically on the milieu regardless of the signals powers which they traverse it.

Subsequently, we will study the polarization-dependent gain (PDG) of SOA for different probe polarizations. This study is based on similar measures to those achieved previously but this time around the probe continuous polarized in either TE or TM.

Figure 7 shows the results obtained for different configurations of polarization of the probe at the entering of the SOA. According to this figure, it is noted that the SOA is relatively insensitive to the polarization when the polarization of the probe is TM (fig.7 (a)) compared to the TE polarization (fig.7 (b)).

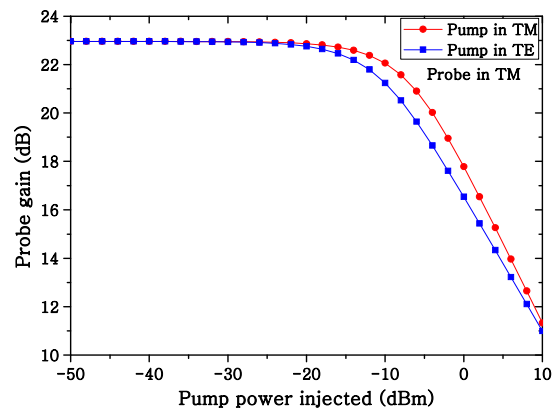

(a)

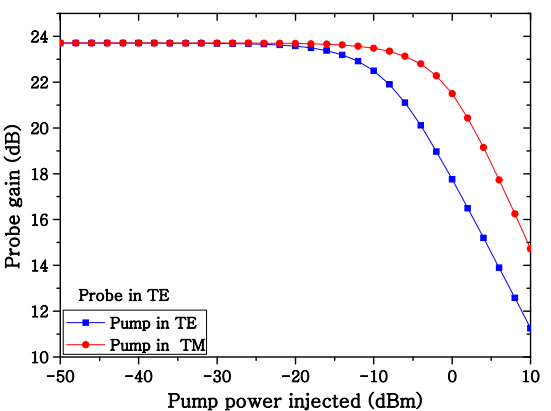

(b)

Figure 7. Measure of the PDG of SOA in different polarization configurations probe. (a) : Probe in TM ; (b) : Probe in TE. The power probe is $-20 \mathrm{dBm}$

The curves show that there isn't a large variation concerning the small signal gain and the gain compression. By contrast, the saturation power of the gain is the only parameter which varies from one configuration of polarization of the pump to another. This is more precisely noted in the equations below:

$$
\begin{aligned}
& \text { Case where the probe is in TM } \\
& \begin{cases}P_{\text {sat }}=1 \mathrm{dBm} & \text { pump in } T E \\
P_{\text {sat }}=0 \mathrm{dBm} & \text { pump in } T M\end{cases}
\end{aligned}
$$

Knowing that using the $\mathrm{SOA}_{\mathrm{s}}$ as nonlinear optical gates must present a strong small signal gain, a low power of saturation as well as a more efficient possible compression with a very steep slope of gain. For this, the configuration of polarization of both the probe and pump in TE is the most suitable for the use of our component as optical gate. The other cases of configuration can be used to improve the basic function of SOA (the amplification).

\section{CONCLUSION}

With the aid of a numerical algorithm based on the finite difference method (FDM), we have studied the static characteristics of the birefringence effect in the SOA.

In our research, we have also included in this method the evolution equation of the carrier density to take into account any change in the bias current and the different carriers' recombinations. To demonstrate the capability of the model, some simulation results of SOA have been presented.

As we have seen, the characteristic of the ASE power enables to determine the maximum of bias current that can support a SOA. The ASE spectra indicate the wavelengths for which the SOA is adapted (around the gain peak). The characterizations of static gain show the small signal gain as well as the saturation power and the slope of the gain curve.

The measure of polarization dependent gain (PDG) of the SOA in self-saturation and crosssaturation shows that the component is intrinsically depending on the polarization. Once the optimum operating parameters and the associated characteristic quantities are determined, the step for specifying whether a SOA is a good candidate for the applications in optical signal processing is the dynamic characterization of the component. 


\section{REFERENCES}

[1] H. Lirong, H. Dexiu, "Spectral broadening of ultrashort optical pulse due to birefringence in semiconductor optical amplifiers”, optics communications, vol. 223, pp. 295-300, 2003.

[2] L.Q. Guo and M.J. Connelly, "A Mueller-Matrix Formalism for modeling Polarization Azimuth and Ellipticity Angle in Semiconductor Optical Amplifiers in Pump-Probe Scheme”, Journal of Lightwave Technology, vol. 25, pp. 410-420, 2007.

[3] O.L. Ladouceur, K. Bergman, "Polarization-dependent gain in SOA-based optical multistage interconnection networks”, Journal of Lightwave Technology, vol. 24, pp. 3959-3967, 2006.

[4] M.R. Rakhshani, M.A. M. Birjandi, "New Design of Channel Drop Filter by Triangular Photonic Crystal", International Journal of Electrical and Computer Engineering (IJECE), vol. 3, no. 1, pp.73-77, 2013.

[5] A.Elyamani, A. Zatni, "steady-state of numerical model and the design of a wideband semiconductor optical amplifier using the finite difference method", Journa of Theoretical and Applied Information technologie, vol. 51, pp. 400-409, 2013.

[6] Dong-Xue Wang, JOHN A. Buck, Kevin Brennan, and Ian Ferguson. "A numerical model of wavelength converters based on cross-gain modulation in semiconductor optical amplifiers”, Applied Optics Engineer, vol. 45, pp. 47014-4708, 2006.

[7] M.J. Connelly, "Wideband Semiconductor Optical Amplifier Steady-state Numerical Model”, IEEE J. Quantum Electronics, vol. 37, pp. 439-447, 2001.

[8] A. Zatni and J. Le Bihan, “Analysis of FM and AM responses of a tunable three-electrode DBR laser diode”, IEEE Journal of Quantum Electronics, vol. 31, pp. 1009-1014, 1995.

[9] JOSEPH DÉSIRÉ TOPOMONDZO, "Etude des effets de biréfringence induite dans les amplificateurs optiques à semi-conducteurs. Applications dans les systèmes de communications optiques,” Thèse de Doctorat (2004).

[10] Mats Gustavsson, "Analysis of Polarization Independent Optical Amplifiers and Filters Based on Polarization Rotation in Periodically Asymmetric Waveguides”, IEEE Journal of Quantum Electronics, vol. 29, no. 4, pp. 11681178, 1993.

[11] Md. Masud Rana, Muhammad Abdul Goffar Khan,"Dense Wavelength Division Multiplexing Optical Network System”, International Journal of Electrical and Computer Engineering (IJECE), vol. 2, no. 2, pp. 203-206, 2012.

\section{BIOGRAPHIES OF AUTHORS}

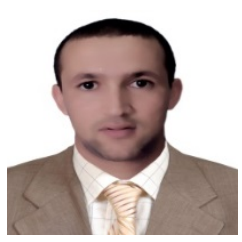

Abdenbi. ELYAMANI was born in Kenitra, Morocco, on January $1^{\text {st }}, 1984$. He received the MSc degree in electrical and electronics system engineering from faculty of sciences University Ibnou Zohr in 2009. He is currently preparing his $\mathrm{PhD}$ at the centre of doctoral studies (Ibnou Zohr CED). His research interests include design, characterization, modelling and optimization of optoelectronic components and fibre optic communications systems.

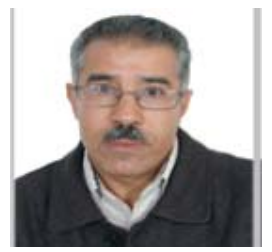

Abdelkarim. ZATNI was educated at the Telecom Bretagne University France; He obtained a $\mathrm{PhD}$ at the National School of Engineers of Brest France in 1994. He has been teaching for 20 years. He is currently a Professor and the Head of computer science department in Ibnou Zohr University at Higher School of technology Agadir, Morocco. He conducts his research and teaches computer science and telecommunications. 